\title{
Is stress related to the presence and persistence of oncogenic human papillomavirus infection in young women?
}

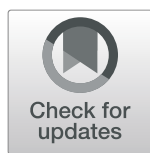

Ulrike Kuebler', Susanne Fischer ${ }^{1}$, Laura Mernone ${ }^{1}$, Christian Breymann$^{2}$, Elvira Abbruzzese ${ }^{1}$ and Ulrike Ehlert ${ }^{1^{*}}$ (D)

\begin{abstract}
Background: Persistent infection with high-risk human papillomavirus (HR-HPV) is the most important risk factor for the development of cervical cancer, but factors contributing to HR-HPV persistence are incompletely understood. The objective of this study was to test for associations of chronic stress and two aspects of diurnal cortisol secretion (i.e., the cortisol awakening response [CAR] and total cortisol output over the day [AUCgday]) with HR-HPV status at baseline and 12 months later (follow-up).

Methods: We evaluated 188 women ( $25 \pm 3$ years) at baseline. Follow-up investigation was restricted to HR-HPV infected women at baseline. Of the initial 48 HR-HPV positive participants, 42 completed the follow-up (16 HR-HPV positive and 26 HR-HPV negative). At baseline and follow-up, we determined HR-HPV status in cervical smears, assessed chronic stress, and repeatedly measured salivary cortisol over the day. At baseline, we analyzed salivary cortisol only in a subgroup of 90 participants (45 HR-HPV negative and 45 HR-HPV positive).
\end{abstract}

Results: At baseline, higher chronic stress (excessive demands at work: $p=.022$, chronic worrying: $p=.032$ ), and a higher CAR $(p=.014)$ were related to baseline HR-HPV positivity. At follow-up, there was a statistical trend for a positive association between the CAR and HR-HPV positivity $(p=.062)$. Neither the CAR nor the AUCgday mediated the associations between chronic stress and HR-HPV status.

Conclusions: Our findings suggest that both chronic stress and diurnal cortisol are related to the presence of HRHPV infection and may thus play a role in HPV-associated cervical carcinogenesis.

Keywords: Oncogenic human papillomavirus, Cervical cancer, Chronic stress, Salivary cortisol

\section{Background}

Cervical cancer is the fourth most common cancer in women worldwide, with an estimated incidence of 500, 000 cases per year $[1,2]$. The most important risk factor for the development of cervical cancer is high-risk human papillomavirus (HR-HPV) infection of cervical mucosa cells [3]. More than $60 \%$ of HR-HPV infections are cleared within 6-12 months and cause no pathological

\footnotetext{
* Correspondence: u.ehlert@psychologie.uzh.ch

'Department of Clinical Psychology and Psychotherapy, University of Zurich, Binzmühlestrasse 14/26, 8050 Zurich, Switzerland

Full list of author information is available at the end of the article
}

changes, but in some women infections persist and can progress to cervical intraepithelial neoplasia (CIN) and cervical cancer $[4,5]$. Although the factors and precise mechanisms regulating the course of HR-HPV infection are not completely understood, clinical and epidemiological data revealed an association between HR-HPV clearance and effector functions of $\mathrm{T}$ lymphocytes, also referred to as cell-mediated immunity (CMI) [6, 7].

Animal and human studies provide converging evidence that chronic stress compromises CMI, particularly cytotoxic $\mathrm{T}$ lymphocyte (CTL) and CD4+ T cell activity, CD4+ T cell and CTL counts, and lymphocyte migration 
and proliferation [8-15]. There is evidence that the stress effects on the immune system are mediated by glucocorticoids (GCs) [9, 16, 17], which are released from the adrenal cortex into plasma as the end product of the hypothalamus-pituitary-adrenal (HPA) axis in response to stress and thus in characteristic stress-related patterns [18]. A large body of literature indicates prolonged hypersecretion of GCs and heightened GC secretion occurring during the first hour after awakening (cortisol awakening response, CAR) under chronic job and general life stress conditions $[19,20]$. Increased GC levels in turn have been shown in vitro and ex vivo to compromise the equilibrium between type $1 \mathrm{~T}$-helper (Th1) cell and Th2 cell activity by differential modulation of cytokine expression, resulting in a shift from a Th1 to a Th2 response [21]. Notably, whereas Th1 cell activity contributes to CMI, Th2 cell activity favors the humoral immune response [22-24]. Hence, increased stress-induced GC levels are presumed to systematically suppress Th1-mediated CMI, while causing a shift toward Th2-mediated humoral immunity.

Considering the suppressive effects of chronic stress and increased GC levels on CMI, and given the importance of cell-mediated immune responses in HR-HPV clearance, chronic psychological stress may participate in promoting $\mathrm{HR}-\mathrm{HPV}$ persistence and thus HPV-associated cervical cancer progression mediated by cortisol. Indeed, several studies have demonstrated associations between psychosocial stress and prevalence and progression of HPVassociated cervical dysplasia and cancer [25-31], although not unequivocally so [32, 33]. Among the hitherto published studies, however, most used cross sectional or retrospective designs and focused on the association between stress and cervical dysplasia or cancer; only two studies were of prospective nature $[31,32]$ and only one directly focused on the association between chronic stress (i.e., bereavement) and HR-HPV infection [25].

Concerning mechanisms that might underlie the possible link between psychosocial stress and HR-HPV persistence, a case-control study in Caucasian women provided first evidence for stress-related decrements in proliferative $\mathrm{T}$-cell responses to $\mathrm{HPV}-16$ suggesting suppressed cell-mediated immune responses as mediator [34]. However, to the best of our knowledge, no study has previously examined associations between cortisol and HR-HPV presence or persistence as an additional psycho-endocrine mechanism linking stress and HPVmediated cervical neoplasia and cancer.

Therefore, the purpose of this study was to test for associations of chronic stress and two aspects of diurnal cortisol secretion (i.e., the cortisol awakening response and total cortisol output over the day), with HR-HPV presence and persistence. We measured chronic stress, salivary cortisol, and HR-HPV status at baseline and 12months after baseline. Our main hypotheses were that a) higher levels of chronic stress, a higher cortisol awakening response, and a higher total cortisol output would be associated with an increased risk of HR-HPV positivity at baseline (i.e., HR-HPV presence) and 12 months after baseline (i.e., HR-HPV persistence) and that b) the hypothesized association between chronic stress and HR-HPV presence/persistence would be statistically mediated by a higher cortisol awakening response and by a higher total cortisol output.

\section{Methods}

\section{Participants}

This study was part of a large research project, in which a random sample of 188 young women was recruited by aid of collaborating physicians and advertisements. Participants with any self-reported acute or chronic immune-related disease (e.g., autoimmune disorder, HIV-positive status, current infectious diseases) were not eligible for the study. Explicit exclusion criteria, obtained by participants' self-report, were: age $<18$ or $>31$ years, virginity, HPV vaccination, pregnancy in the past or currently pregnant, irregular gynecological check-ups (i.e., more than 2 years in between visits, representing the standard in Switzerland), HPV diagnosis at last gynecological check-up, treatment of cervical abnormalities during the last year, and previous cervical conization.

Of the total of 1477 participants assessed for study eligibility, 188 participants were enrolled (see Fig. 1 for details on participant flow). Of these, 48 participants were tested HR-HPV positive and $140 \mathrm{HPV}$ negative. Based on a power analysis (see statistical analysis) and for reasons of limited funding, we analyzed salivary cortisol in 90 participants (i.e., $45 \mathrm{HR}-\mathrm{HPV}$ negative [cortisol-control group] and 45 HR-HPV positive). Notably, three participants of the HR-HPV positive group had to be excluded because of non-adherence to sampling time (see study procedure). The allocation of HR-HPV negative participants to the 'cortisol-control group' $(n=45)$ was randomly achieved by using 140 opaque and sealed envelopes for all of our $140 \mathrm{HR}-\mathrm{HPV}$ negative participants, i.e., one envelope for each HRHPV negative participant. An independent research assistant generated the random allocation sequence by sealing, mixing, and subsequently opening 45 opaque envelopes. Due to technical problems with assaying, data of cortisol were missing in 4 participants rendering a final sample of $41 \mathrm{HR}-\mathrm{HPV}$ negative women and $45 \mathrm{HR}$ HPV positive women for cortisol measurements.

Follow-up investigation was restricted to women with HR-HPV infection at baseline. Of the initial $48 \mathrm{HR}-\mathrm{HPV}$ positive participants, $42(87.5 \%)$ completed the 12-month 


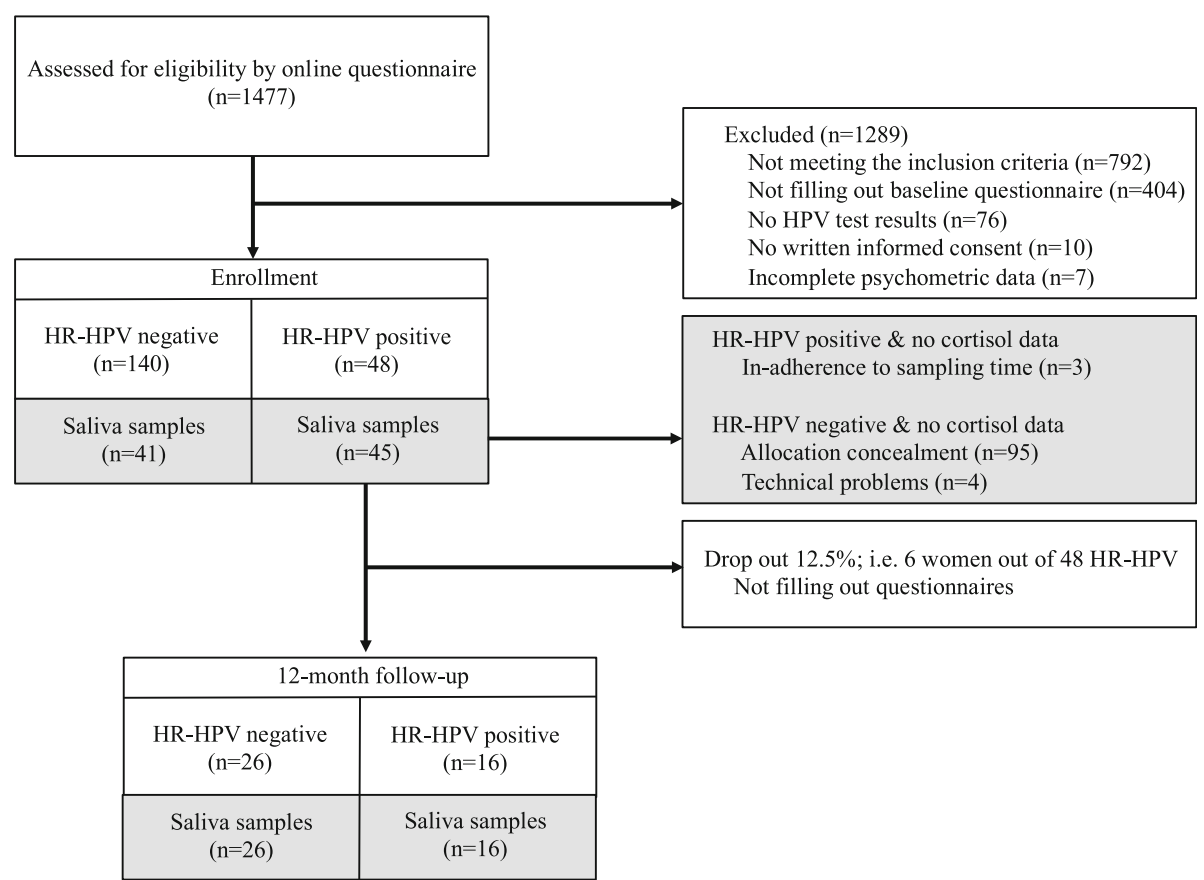

Fig. 1 Participant flow throughout the study

follow-up. Of these, 16 participants (38\%) were still HRHPV positive while 26 participants (62\%) were negative to HR-HPV.

\section{Procedures}

All participants who passed the screening were given detailed written information about the study purpose and procedure. Two weeks before their next routine check-up with their gynecologist, the participants received a link for a baseline online questionnaire (T1), which included questions addressing demographics, chronic stress, and suspected covariates (see selfreported measures for more information). The questionnaire had to be filled in before the routine gynecological check-up. In addition, participants received material (DNAPap $^{\text {TM }}$ Cervical Sampler; Qiagen Gaithersburg, Inc., USA) and written instructions for HPV testing by means of a cervical swab, which was to take place during the routine check-up at their gynecologist's practice. In detail, participants were asked not to schedule their routine gynecological check-up during menstruation, to abstain from vaginal cream, spermicides, and lubricants three days before the examination, and to abstain from sexual intercourse two days before the examination. Cervical samples were collected by healthcare professionals during the routine gynecological check-up. After cervical cells were collected via the DNAPap ${ }^{\mathrm{TM}}$ Cervical Sampler, the system was sent to a specialized laboratory for HPV testing (Laboratory Enders \& Partners; Stuttgart, Germany).
Moreover, participants received material and written instructions for saliva collection to assess the diurnal cortisol secretion using Salivette collection devices (Sarstedt, Sevelen, Switzerland). All participants were instructed to collect 7 saliva samples over the course of a regular workday within two weeks after HPV testing. For cortisol awakening response assessment, saliva samples were collected immediately after awakening (while still lying in bed), 30, and $60 \mathrm{~min}$ thereafter. To assess the cortisol output over the day, samples were collected at 11:00, 14:00, 17:00, and 20:00 h. Participants were asked to abstain from strenuous physical activity and from alcohol consumption $24 \mathrm{~h}$ before saliva collection. In addition, participants were asked to abstain from smoking starting on the morning of saliva collection as well as from brushing their teeth, eating and drinking for $20 \mathrm{~min}$ prior to sample collection. To control the sampling time self-reports of the exact sampling time were used. Three participants of the HR-HPV positive group had to be excluded from the analysis due to sampling time. Saliva samples were stored in a fridge until sampling was completed and were then sent to the biochemical laboratory at the Institute of Psychology of the University of Zurich, Switzerland, and stored at - 20 $\mathrm{C}$ until biochemical analysis.

Follow-up investigation was restricted to women with HR-HPV infection at baseline. For follow-up 12 months after baseline (T2), participants with HR-HPV positive status at baseline were contacted again 1 month before 
T2. Notably, we chose a follow-up of 12 months because the risk of CIN grade 2 or worse diagnosis by 30 months has been found to be over $50 \%$ among women younger than 30 years with HR-HPV infections that persisted at least 12 months [4]. Study procedure as well as psychometric and biochemical measures were analogous to T1. In brief: Participants had to fill in an online questionnaire within a period of 2 weeks before a cervical swab. In addition, they were instructed to collect 7 saliva samples over the course of a regular workday within a period of two weeks after cervical swab.

The Ethics Committee of the University of Zurich, Switzerland, formally approved the research protocol, which was conducted in accordance with the principles of the Declaration of Helsinki. All participants provided written informed consent before participating in the study and were compensated with $120 \mathrm{CHF}$ for their participation.

\section{Biological measures \\ Assessment of HPV status}

Cervical swab cells collected with the DNAPap ${ }^{\text {тм }}$ Cervical Sampler were tested for HR-HPV infections at the laboratory Enders \& Partners (Stuttgart, Germany) using the hc2 High-Risk HPV DNA Test ${ }^{\oplus}$ from QIAGEN (Gaithersburg, Inc., USA). The hc2 is an in vitro nucleic acid hybridization assay with signal amplifications using microplate chemiluminescence for the qualitative detection of DNA in cervical specimens of 13 of the most frequently found HR-HPV types in cervical tumors [35], i.e., HR-HPV 16, 18, 31, 33, 35, 39, 45, 51, 52, 56, 58, 59, 68. The assay was performed with cutoff of $1.0 \mathrm{pg} / \mathrm{ml}$ recommended by Food and Drug Administration (FDA).

\section{Cortisol analysis}

At baseline, we analyzed salivary cortisol only in a subgroup of 90 participants (i.e., $45 \mathrm{HR}-\mathrm{HPV}$ negative and 45 HR-HPV positive) due to reasons of limited funding (see section study participants). Thawed saliva samples were centrifuged at $2000 \mathrm{~g}$ for $10 \mathrm{~min}$, yielding low-viscosity saliva. Salivary free cortisol concentrations were determined using a commercial chemiluminescence immunoassay with high sensitivity of $0.16 \mathrm{ng} / \mathrm{ml}$ (LIA, IBL Hamburg, Germany). Inter- and intra-assay variation was below 11.5 and $7.7 \%$, respectively. All samples of one subject were analyzed in the same run.

\section{Psychological measures \\ Chronic stress}

We assessed the amount of chronic stress over the last 3 months both at baseline and follow-up and by means of the Trier Inventory for the Assessment of Chronic Stress (TICS) [36]. The TICS is a 57 -item questionnaire measuring 9 interrelated factors of chronic stress in addition to a screening scale for chronic stress (SSCS): 1) Work Overload, 2) Social Overload, 3) Pressure to Perform, 4) Work Discontent, 5) Excessive Demands at Work, 6) Lack of Social Recognition, 7) Social Tensions, 8) Social Isolation, and 9) Chronic Worrying. Using a 5point rating scale ranging from 0 (never) to 4 (very often), participants were asked to rate how often they have had a certain experience or have experienced a certain situation within the last three months. Higher scores mean higher extent of chronic stress. Cronbach's alpha was .87 in our sample for the SSCS indicating good reliability.

\section{Control variables}

We assessed contraceptive use, age at onset of sexual intercourse, total number of sexual partners, and smoking status and intensity based on previous findings suggesting associations with risk of genital HPV infection [37]. Smoking intensity was assessed by the average number of monthly smoked cigarettes. The total number of sexual partners was assessed by asking the participants how many sexual partners they have had in the past. Hormonal contraceptives were binary coded as using hormonal contraceptives vs. not using contraceptives. Moreover, we assessed sleep quality using the Pittsburgh Sleep Quality Index (PSQI) [38] due to its potential effects on cortisol responses [39, 40].

\section{Statistical analyses}

Data were analyzed using SPSS Inc. version 23.0 for Mac OS X (Chicago, IL, USA) and presented as mean \pm SEM. $\mathrm{G}^{*}$ Power 3.1 defined an optimal sample size of $n=90$ to detect a medium effect size of $\mathrm{f}=.30$ in general linear models given two groups and the cortisol awakening response (CAR) or the daily area under the curve with respect to the ground (AUCgday [41]), respectively, as dependent variable with a power of 0.80 . All tests were two-tailed with the significance level set at $p \leq .05$ and the level of a statistical trend set at $p \leq .10$. Data were tested for normal distribution using a KolmogorovSmirnov test before further statistical procedures were applied. We applied Huynh-Feldt correction for repeated measures. The CAR was calculated as the difference between the peak cortisol response (i.e., $30 \mathrm{~min}$ after awakening) minus cortisol level at awakening [42]. To evaluate total cortisol output over the study period, the AUCgday was calculated integrating the respective 0 min, $30 \mathrm{~min}$, and $60 \mathrm{~min}$ after awakening, 11:00, 14:00, 17:00, and 20:00 cortisol sample. Body mass index (BMI) was calculated as the ratio of weight in kilograms to height in square meters. Across the study groups univariate analyses of variance (ANOVAs) were calculated to test for differences in group characteristics and chronic stress. To test for associations between chronic stress 
and HR-HPV status, logistic regression analyses were performed. To predict HR-HPV status at T1 or T2, we entered in a first step (model 1) potential HR-HPV risk factors (covariates) and in a second step (model 2) the chronic stress factor.

To examine the associations between cortisol and HRHPV status, repeated measures ANCOVAs were applied with HR-HPV status as the group variable and the 3 (CAR) or 7 (AUCgday) time points at which cortisol was measured as repeated measures. To additionally test for group differences in the CAR and in the AUCgday, and single cortisol time points, we performed univariate ANCOVAs. To examine whether the cortisol measures (CAR, AUCgday) were mediators of the hypothesized associations between chronic stress and HR-HPV status, we performed mediator analyses using the PROCESS macro [43]. In the proposed mediation model chronic stress was tested as predictor and the HR-HPV status as outcome with the cortisol measures (i.e., CAR, AUCgday) as mediators. Statistical mediation of an association between predictor and outcome holds if the predictor is significantly associated with the mediator, and the mediator is significantly associated with the outcome while controlling for the predictor [44]. By default, the PROCESS macro uses a bootstrapping approach with 1000 bootstrap resamples and a 95\% confidence interval of the indirect effects, which were adopted in this study.

In terms of covariates, we controlled a priori for the potential HPV risk factors contraceptive use, age at onset of sexual intercourse, number of sexual contacts, and smoking intensity [37] in all statistical analyses involving HR-HPV status at baseline and follow-up. Additionally, we have taken sleep quality into account as covariate in all statistical analyses involving cortisol measures. Effect size parameters $(f)$ were calculated from partial $\eta^{2}$-values and are reported where appropriate (effect size conventions: $f: .10=$ small, $.25=$ medium, $.40=$ large).

\section{Results}

\section{Participant characteristics}

Table 1 (left) provides the characteristics of participants per HR-HPV status at T1 (baseline). The HR-HPV positive group $(n=48)$ had more sexual partners during their lifetime and included more smokers than the HR-

Table 1 Participants' characteristics per HR-HPV status at baseline and at follow-up

\begin{tabular}{|c|c|c|c|c|c|c|c|c|}
\hline & \multicolumn{3}{|l|}{ HR-HPV at baseline } & \multirow{2}{*}{$\begin{array}{l}P \text {-value } \\
1 \text { vs. } 3\end{array}$} & \multirow[b]{2}{*}{2 vs. 3} & \multicolumn{2}{|c|}{ HR-HPV at follow-up } & \multirow[t]{2}{*}{$P$-value } \\
\hline & $\begin{array}{l}\text { No }(n=140 ; \\
\text { control group; } 1)\end{array}$ & $\begin{array}{l}\text { No }(n=41 ; \\
\text { cortisol-control } \\
\text { group; } 2)\end{array}$ & Yes $(n=48 ; 3)$ & & & No $(n=26)$ & Yes $(n=16)$ & \\
\hline Age (years), $M \pm S E M$ & $24.9 \pm .26$ & $23.8 \pm .44$ & $25.1 \pm .44$ & .63 & .043 & $26.4 \pm .62$ & $26.2 \pm .71$ & .81 \\
\hline BMI $\left(\mathrm{kg} / \mathrm{m}^{2}\right), \mathrm{M} \pm \mathrm{SEM}$ & $21.8 \pm .25$ & $21.2 \pm .37$ & $21.6 \pm .51$ & .73 & .55 & $21.6 \pm .63$ & $21.0 \pm .51$ & .50 \\
\hline Employment, $n$ (\%) & & & & .77 & .25 & & & .17 \\
\hline Yes & $105(75 \%)$ & $27(65.9 \%)$ & 37 (77.1\%) & & & $23(88.5 \%)$ & $16(100 \%)$ & \\
\hline No & $35(25 \%)$ & $14(34.1 \%)$ & $11(22.9 \%)$ & & & $3(11.5 \%)$ & 0 & \\
\hline $\begin{array}{l}\text { Hormonal contraceptive } \\
\text { use, } n(\%)\end{array}$ & $87(62.2 \%)$ & $26(63 \%)$ & $32(67 \%)$ & .58 & .75 & $17(65 \%)$ & $10(62 \%)$ & .85 \\
\hline $\begin{array}{l}\text { Age at onset of sexual } \\
\text { intercourse, } \\
M \pm S E M\end{array}$ & $17.1 \pm .17$ & $17.2 \pm .33$ & $16.6 \pm .27$ & .13 & .15 & $16.3 \pm .35$ & $16.8 \pm .46$ & .42 \\
\hline Marital status, n (\%) & & & & .86 & .90 & & & .063 \\
\hline Single & 38 (27.1\%) & 7 (17.1\%) & $18(37.5 \%)$ & & & $8(30.8 \%)$ & $2(12.5 \%)$ & \\
\hline Relationship & 95 (67.9\%) & $34(82.9 \%)$ & $26(54.2 \%)$ & & & $18(69.2 \%)$ & $12(75 \%)$ & \\
\hline Married & $7(5 \%)$ & 0 & $3(6.2 \%)$ & & & 0 & $2(12.5 \%)$ & \\
\hline Widowed & 0 & 0 & $1(2.1 \%)$ & & & 0 & 0 & \\
\hline $\begin{array}{l}\text { Total number of } \\
\text { sexual partners, } \\
\mathrm{M} \pm \text { SEM }\end{array}$ & $7.1 \pm 7.4$ & $5.5 \pm 5.6$ & $10.8 \pm 8.4$ & .005 & .001 & $13.9 \pm 13.1$ & $8.9 \pm 6.0$ & .16 \\
\hline Smoker, n (\%) & 43 (30.7\%) & $15(36.6 \%)$ & 25 (52.1\%) & .008 & .143 & $17(65.4 \%)$ & $5(31.3 \%)$ & .075 \\
\hline $\begin{array}{l}\text { Cigarettes per month, } \\
\mathrm{M} \pm \text { SEM }\end{array}$ & $106.4 \pm 152.7$ & $64.7 \pm 116.2$ & $128.1 \pm 111.5$ & .54 & .095 & $128.8 \pm 125.1$ & $99.2 \pm 77.6$ & .63 \\
\hline $\begin{array}{l}\text { Sleep quality (PSQI), } \\
M \pm S E M\end{array}$ & $9.55 \pm 1.93$ & $9.39 \pm 2.21$ & $9.96 \pm 2.13$ & .23 & .23 & $4.77 \pm 2.77$ & $4.25 \pm 1.69$ & .50 \\
\hline
\end{tabular}


HPV negative control group $(n=140)$. No significant group differences were found in terms of age, BMI, employment, marital status, use of hormonal contraceptives, age at onset of sexual intercourse, sleep quality and numbers of cigarettes smoked per month. When comparing the HR-HPV positive group with the HRHPV negative cortisol-control group $(n=41)$ the results did not significantly change, with the exception of age and numbers of cigarettes smoked per month. The HRHPV positive group was older and engaged in heavier cigarette smoking when compared to the HR-HPV negative cortisol-control group. At T2 (follow-up), the HRHPV positive group $(n=16)$ included less smokers and single women than the HR-HPV negative group $(n=26)$. No group differences were found in terms of age, BMI, employment, use of hormonal contraceptives, age at onset of sexual intercourse, number of cigarettes smoked per month, and sleep quality; see Table 1 [right]). See Table 2 for participants' psychological characteristics per HR-HPV status at T1 and T2.

\section{Associations between chronic stress and HR-HPV status}

We calculated logistic regression analyses to address whether chronic stress was associated with the likelihood of being HR-HPV positive at T1 and T2. At baseline, higher levels of excessive demands at work were significantly related to HR-HPV positivity $(b=.143, p=.022$, $\mathrm{OR}=1.15,95 \% \mathrm{CI}: 1.02-1.30$ ), independent of contraceptive use, age at onset of sexual intercourse, number of sexual contacts, and smoking intensity. Moreover, higher levels of chronic worrying $(b=.152, p=.032$, $\mathrm{OR}=1.16,95 \% \mathrm{CI}: 1.01-1.34$ ) at $\mathrm{T} 1$ were related to HRHPV positivity at $\mathrm{T} 1$, independent of contraceptive use, age at onset of sexual intercourse, number of sexual contacts, and smoking intensity. At follow-up, chronic stress levels remained the same (except for a slight increase in lack of social recognition, $p=.025$ ), but no significant associations with the likelihood of being HR-HPV positive emerged.

\section{Associations between cortisol and HR-HPV status}

At baseline, the study groups significantly differed in post-awakening cortisol levels (interaction group-bytime: $\mathrm{F}(1.9 / 144.1)=3.53, p=.035, \mathrm{f}=0.21$; see Fig. $2 \mathrm{a})$, independent of contraceptive use, age at onset of sexual intercourse, number of sexual contacts, smoking intensity, and sleep quality. Post hoc tests revealed no significant group differences in cortisol levels at awakening, but a significantly higher cortisol peak response to awakening in the HR-HPV positive group compared to the HR-HPV negative group (F $(1 / 77)=6.31, p=.014, \mathrm{f}=$ 0.29 ). The groups also significantly differed in cortisol output over the day (interaction group-by-time: F (3.0/ 233.7 ) $=3.41, p=.018, \mathrm{f}=0.21$ ), with a higher (integrated) overall cortisol secretion in the HR-HPV positive group as compared to the HR-HPV negative group by trend (AUCgday: $\mathrm{F}(1 / 77)=3.714, p=.058, \mathrm{f}=0.22$ ).

At follow-up, the study groups significantly differed in post-awakening cortisol levels (interaction group-bytime: $\mathrm{F}(2 / 70)=3.93, p=.024, \mathrm{f}=0.34$; see Fig. $2 \mathrm{~b})$, independent of contraceptive use, age at onset of sexual intercourse, number of sexual contacts, smoking intensity, and sleep quality. Post hoc tests revealed no significant group differences in cortisol level at awakening. However, on a trend level, the HR-HPV positive group

Table 2 Participants' psychological characteristics per HR-HPV status at baseline and at follow-up given as means and standard errors

\begin{tabular}{|c|c|c|c|c|c|c|c|c|}
\hline & \multicolumn{3}{|c|}{ HR-HPV at baseline (T1) } & \multicolumn{2}{|c|}{$P$-value } & \multicolumn{2}{|c|}{ HR-HPV at follow-up (T2) } & \multirow[t]{2}{*}{$P$-value } \\
\hline & $\begin{array}{l}\text { No }(n=140 ; \\
\text { control group) }\end{array}$ & $\begin{array}{l}\text { No }(n=41 ; \\
\text { cortisol-control } \\
\text { group) }\end{array}$ & Yes $(n=48)$ & 1 vs. 3 & 2 vs. 3 & No $(n=26)$ & Yes $(n=16)$ & \\
\hline \multicolumn{9}{|l|}{ TICS } \\
\hline Work overload & $21.4 \pm 0.50$ & $21.2 \pm 1.08$ & $22.5 \pm 0.94$ & .30 & .37 & $23.4 \pm 1.56$ & $23.9 \pm 1.82$ & .82 \\
\hline Social overload & $14.1 \pm 0.36$ & $13.0 \pm 0.68$ & $14.1 \pm 4.9$ & .99 & .30 & $16.1 \pm 1.06$ & $15.8 \pm 1.08$ & .84 \\
\hline Pressure to perform & $24.6 \pm 0.46$ & $23.0 \pm 0.86$ & $24.1 \pm 0.82$ & .59 & .34 & $26.2 \pm 1.07$ & $24.4 \pm 1.32$ & .32 \\
\hline Work discontent & $18.2 \pm 0.42$ & $17.3 \pm 0.80$ & $19.1 \pm 0.75$ & .29 & .11 & $19.0 \pm 1.00$ & $19.4 \pm 1.01$ & .78 \\
\hline Excessive demands at work & $11.8 \pm 0.28$ & $10.9 \pm 0.55$ & $13.1 \pm 0.66$ & .044 & .017 & $12.7 \pm 0.88$ & $12.2 \pm 1.05$ & .74 \\
\hline Lack of social recognition & $8.7 \pm 0.30$ & $8.1 \pm 0.5$ & $8.4 \pm 0.41$ & .53 & .67 & $9.1 \pm 0.62$ & $9.3 \pm 0.63$ & .83 \\
\hline Social tensions & $11.4 \pm 0.30$ & $10.8 \pm 0.48$ & $10.7 \pm 0.48$ & .29 & .97 & $11.3 \pm 0.74$ & $13.1 \pm 1.12$ & .18 \\
\hline Social isolation & $12.7 \pm 0.40$ & $12.0 \pm 0.82$ & $13.1 \pm 0.72$ & .68 & .35 & $12.6 \pm 1.00$ & $13.3 \pm 1.42$ & .69 \\
\hline Chronic worrying & $10.7 \pm 0.28$ & $9.7 \pm 0.57$ & $11.4 \pm 0.57$ & .29 & .042 & $10.7 \pm 0.82$ & $12.3 \pm 1.10$ & .25 \\
\hline Screening scale (SSCS) & $28.8 \pm 0.59$ & $26.8 \pm 1.35$ & $30.3 \pm 1.26$ & .21 & .061 & $30.2 \pm 1.99$ & $32.1 \pm 2.17$ & .54 \\
\hline
\end{tabular}




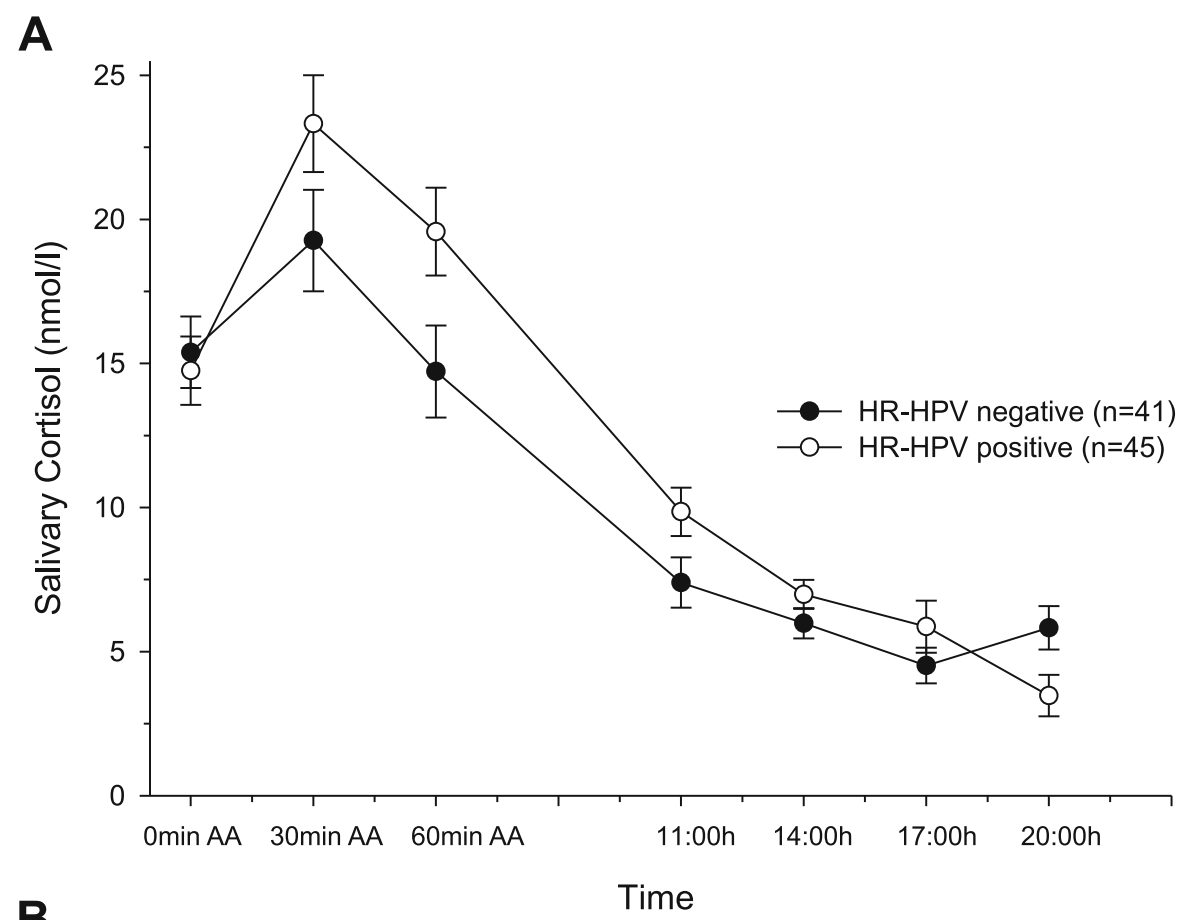

B

Time

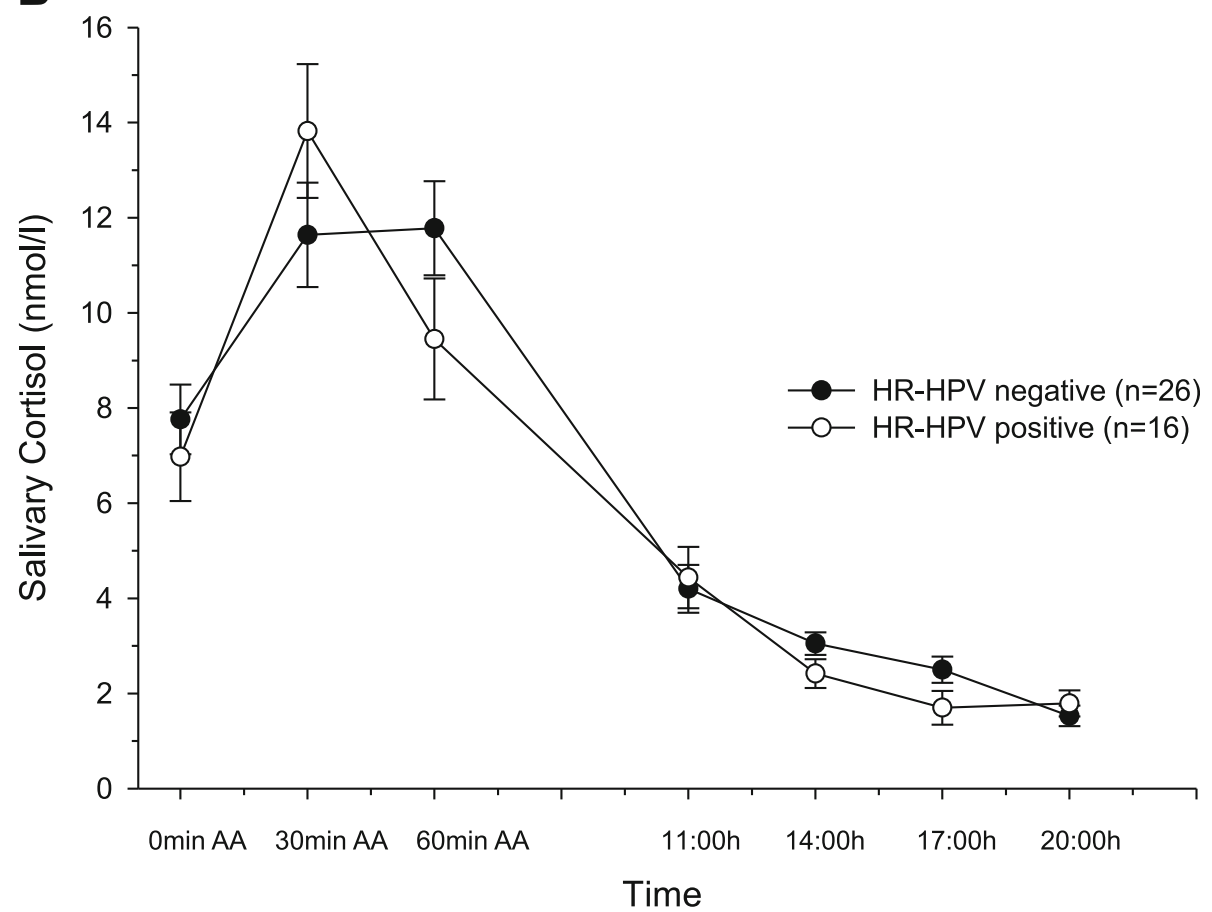

Fig. $\mathbf{2}$ a \& $\mathbf{b}$ Cortisol awakening response and cortisol output over the day in women with and without HR-HPV given as means and standard errors. AA, after awakening. a: At baseline. b: At follow-up

showed a higher cortisol peak response to awakening than the HR-HPV negative group (F $(1 / 35)=3.71), p=$ $.062, \mathrm{f}=0.33)$. The cortisol output over the day did not differ significantly between the HR-HPV positive group and the HR-HPV negative group (interaction effect group-by-time: $p=.09$; AUCgday: $p=.50$ ).

\section{Mediation of stress chronic stress effects on HR-HPV} status by cortisol

Neither the CAR nor the AUCgday were found to mediate the associations between chronic stress (i.e., excessive demands at work and chronic worrying) and HR-HPV status at T1. 


\section{Discussion}

To the best of our knowledge, this is the first study that investigated the effects of chronic stress and different aspects of diurnal cortisol secretion on HR-HPV presence and persistence in young women.

As hypothesized, we found higher chronic stress levels, as measured by the TICS subscales excessive demands at work and chronic worrying to be associated with increased likelihood of HR-HPV positivity at baseline. These results were independent of potential HPV infection risk factors that were assessed at each measurement time point, including number of sexual contacts, age at onset of sexual intercourse, smoking intensity, and contraceptive use.

Our results provide first indications that chronic stress is an independent risk factor for HR-HPV presence in young women. This finding is in line with the hitherto single published human study that investigated associations between HPV infection and psychosocial stress. In this large-scale case-control study, bereaved women (i.e., loss of a family member due to death) showed a $62 \%$ increased risk of HR-HPV prevalence [25], but analyses were not adjusted for potential HPV infection risk factors. Thus, the independent effect of bereavement remains unclear. In light of the substantially increased risk of CIN grade 2 or worse diagnosis among women younger than 30 years with HR-HPV infections that persisted at least 12 months [4], our findings may suggest that in particular young women with chronic exposure to stressful demands are susceptible to HPV-associated CIN and cervical cancer. This assumption is strengthened by previous studies that have demonstrated a link between different kind of stressors, including life stressors [29, 31], intimate partner violence [28], passive/helplessness reactions to stress [27], parental death during childhood [26], bereavement [25], and the long-term consequences of persistent HR-HPV infections, i.e., cervical dysplasia and cancer. Notably, the groups did not differ in chronic stress levels at follow-up, which is most likely due to the reduction in the sample size (i.e., from $N=188$ to $n=42$ ). Future studies with longer follow-up periods are needed to verify the proposed role of chronic stress in HPVassociated cervical carcinogenesis.

Regarding mechanisms underlying the link between chronic stress and HR-HPV presence and persistence, our study is the first to investigate different aspects of diurnal cortisol secretion (i.e., CAR and AUCgday) as potential mediators for the association between stress and HR-HPV infection. As hypothesized, we found an increased cortisol awakening response and total cortisol output over the day in HR-HPV positive women compared to HR-HPV negative women at baseline. Likewise, at 12-months follow up, HR-HPV positive women also showed a slightly increased cortisol awakening response compared to HR-HPV negative women. Notably, the groups did not differ in their total cortisol output, which, again, is most likely due to the reduced sample size at 12-months follow up. Group differences were of medium to large effect size and independent of potential HPV infection risk factors. These findings provide first evidence for HPA axis hyperactivity in HR-HPV positive women. Considering the suppressive effects of increased cortisol levels on cell-mediated immune responses [21] that are important in HR-HPV clearance [6, 45] our results also support the suggestion of an active role for cortisol in the control of HR-HPV infection.

Interestingly, however, we were unable to confirm either cortisol awakening response or total cortisol output as mediators of the associations found between chronic stress and HR-HPV presence. For explanation of the missing mediation effect of cortisol awakening response regarding the chronic stress-HR-HPV relationship, we suggest three hypotheses. First, chronic stress and the CAR/diurnal cortisol output were measured on a different time scale (past 3 months vs. present day), which may explain the lack of association. Further studies employing long-term measures of HPA axis functioning (e.g., hair cortisol) may be more likely to uncover significant relationships. Second, the interaction between chronic stress, endocrine, and immune mechanisms in the field of HR-HPV infection might be too complex to be adequately covered by linear statistical models. Finally, it is possible that we were unable to detect a mediation effect of cortisol on the chronic stress-HRHPV associations because our study was underpowered to reveal small to medium effects.

Our results may have clinical implications in that they suggest that stress management programs of young HRHPV positive women with HPV axis hyperactivity and chronic exposure to stress could provide a psychobiological benefit. If stress management programs are directed at helping HR-HPV positive women to adaptively cope with demands of daily life, daily cortisol secretion and the course of HR-HPV infection might be positively affected. For example, it has previously been demonstrated that a stress management program using a combination of educative and cognitive-behavioral skills led to lower chronic stress and cortisol awakening response in participants suffering from chronic stress due to overwork [46].

The present study has several strengths, including the prospective study design that allowed us to investigate the role of chronic stress and cortisol in the course of HR-HPV infection. Moreover, HR-HPV test results were given to the participants after they had completed the study questionnaires. This is important to exclude confounding effects of distress due to being tested HR-HPV positive $[47,48]$ on the stress measures in our study. 
Furthermore, we controlled for a variety of potential HR-HPV risk factors to rule out confounding influence on the investigated associations. The study also has some limitations. It was an observational study, so that causal interpretations of the observed associations remain speculative. The generalizability of our findings might be limited to young women. Furthermore, because of the relatively small sample size and the follow-up period restricted to 12 months our data should be interpreted with caution until confirmed in larger studies with longer follow-up periods. Additionally, only the initially HR-HPV positive individuals were investigated at follow-up. It is possible that women with a negative HR-HPV status at baseline became infected at follow-up. Moreover, condom use has not been assessed in this study, and it cannot be ruled out that our cases with persistent infections may, in fact, have been cases with re-infections. Finally, the reduction of sample size for cortisol assessment in the HR-HPV negative group due to limited financial and organizational reasons must be mentioned as limitation.

\section{Conclusions}

Taken together, we found first evidence that chronic stress and a dysregulated diurnal cortisol secretion might be independent risk factors for HR-HPV presence in young women. Future research is needed to replicate our findings in larger populations with longer follow-up periods.

\begin{abstract}
Abbreviations
ANCOVA: Analyses of covariance; ANOVA: Analysis of variance; AUCg: Area under the curve with respect to ground; BMI: Body mass index; CAR: Cortisol awakening response; CIN: Cervical intraepithelial neoplasia; CMI: Cellmediated immunity; CTL: Cytotoxic T lymphocyte; CVs: Inter- and intra coefficients of variation; GCs: Glucocorticoids; HPA: Hypothalamus-pituitaryadrenal; HR-HPV: High-risk human papillomavirus; PSQl: Pittsburgh Sleep Quality Index; SSCS: Screening scale for chronic stress; Th1: Type 1 T-helper; TICS: Trier Inventory for the Assessment of Chronic Stress
\end{abstract}

\section{Acknowledgements}

We would like to thank all participants involved in our study.

\section{Authors' contributions}

All authors have made substantial contributions to this work. UE and UK conceived and designed the study; CB and EA were also involved in the study project and acquisition of data. UE, UK, SF and LM have analyzed and interpreted the data, and drafted the work or substantively revised it. CB and EA commented on previous versions of the manuscript. All authors read and approved the final manuscript.

\section{Funding}

This study was supported by Oncosuisse (Krebsliga Schweiz).

\section{Availability of data and materials}

The dataset generated and analysed during the current study are not publicly available but are available from the corresponding author on reasonable request.

\section{Declarations}

Ethics approval and consent to participate

The Ethics Committee of the University of Zurich, Switzerland, formally approved the research protocol, which was conducted in accordance with the principles of the Declaration of Helsinki. All participants provided written informed consent before participating in the study.

\section{Consent for publication}

Not applicable.

\section{Competing interests}

The authors declare that they have no competing interests.

\section{Author details}

'Department of Clinical Psychology and Psychotherapy, University of Zurich, Binzmühlestrasse 14/26, 8050 Zurich, Switzerland. ${ }^{2}$ University Hospital Zurich, Zurich, Switzerland.

Received: 13 January 2020 Accepted: 4 March 2021

Published online: 16 April 2021

\section{References}

1. WHO. GLOBOCAN 2018: Estimated cancer indicidence, mortality and prevalence worldwide in 2018 https://gco.iarc.fr/today/data/factsheets/ca ncers/23-Cervix-uteri-fact-sheet.pdf. 2018

2. Serrano B, Brotons M, Bosch FX, Bruni L. Epidemiology and burden of HPVrelated disease. Best Pract Res Clin Obstet Gynaecol. 2018;47:14-26.

3. de Sanjose S, Quint WGV, Alemany L, Geraets DT, Klaustermeier JE, Lloveras $B$, et al. Human papillomavirus genotype attribution in invasive cervical cancer: a retrospective cross-sectional worldwide study. Lancet Oncol. 2010; 11(11):1048-56.

4. Rodriguez AC, Schiffman M, Herrero R, Wacholder S, Hildesheim A, Castle $P E$, et al. Rapid clearance of human papillomavirus and implications for clinical focus on persistent infections. J Natl Cancer Inst. 2008;100(7):513-7.

5. Schiffman M, Castle PE, Jeronimo J, Rodriguez AC, Wacholder S. Human papillomavirus and cervical cancer. Lancet. 2007;370(9590):890-907.

6. Song D, Li H, Li H, Dai J. Effect of human papillomavirus infection on the immune system and its role in the course of cervical cancer. Oncol Lett. 2015;10(2):600-6.

7. Stanley M. Immune responses to human papillomavirus. Vaccine. 2006;24: S16-22.

8. Bonneau RH, Sheridan JF, Feng NG, Glaser R. Stress-induced effects on cellmediated innate and adaptive memory components of the murine immune response to herpes simplex virus infection. Brain Behav Immun. 1991;5(3): 274-95.

9. Dhabhar FS. Effects of stress on immune function: the good, the bad, and the beautiful. Immunol Res. 2014;58(2-3):193-210.

10. Glaser R, MacCallum RC, Laskowski BF, Malarkey WB, Sheridan JF, KiecoltGlaser JK. Evidence for a shift in the Th-1 to Th-2 cytokine response associated with chronic stress and aging. J Gerontol A Biol Sci Med Sci. 2001;56(8):M477-82.

11. Kiecolt-Glaser JK, Glaser R, Gravenstein S, Malarkey WB, Sheridan J. Chronic stress alters the immune response to influenza virus vaccine in older adults. Proc Natl Acad Sci U S A. 1996;93(7):3043-7.

12. Schmidt D, Reber SO, Botteron C, Barth T, Peterlik D, Uschold N, et al. Chronic psychosocial stress promotes systemic immune activation and the development of inflammatory Th cell responses. Brain Behav Immun. 2010; 24(7):1097-104.

13. Dhabhar FS, Malarkey WB, Neri E, McEwen BS. Stress-induced redistribution of immune cells_from barracks to boulevards to battlefields: a tale of three hormones - Curt Richter award winner. Psychoneuroendocrinology. 2012; 37(9):1345-68.

14. Morey JN, Boggero IA, Scott AB, Segerstrom SC. Current directions in stress and human immune function. Curr Opin Psychol. 2015;5:13-7.

15. Segerstrom SC, Miller GE. Psychological stress and the human immune system: a meta-analytic study of 30 years of inquiry. Psychol Bull. 2004;130: 601-30.

16. Dhabhar FS. Enhancing versus suppressive effects of stress on immune function: implications for immunoprotection and immunopathology. Neuroimmunomodulation. 2009;16(5):300-17. 
17. Cain DW, Cidlowski JA. Immune regulation by glucocorticoids. Nat Rev Immunol. 2017;17:233

18. Glaser R, Kiecolt-Glaser JK. Stress-induced immune dysfunction: implications for health. Nat Rev Immunol. 2005;5(3):243-51.

19. Miller GE, Chen E, Zhou ES. If it goes up, must it come down? Chronic stress and the hypothalamic-pituitary-adrenocortical axis in humans. Psychol Bull. 2007;133:25-45

20. Chida Y, Steptoe A. Cortisol awakening response and psychosocial factors: a systematic review and meta-analysis. Biol Psychol. 2009;80(3):265-78.

21. Elenkov IJ. Glucocorticoids and the Th1/Th2 balance. Ann N Y Acad Sci. 2004;1024:138-46

22. Mullen AC, High FA, Hutchins AS, Lee HW, Villarino AV, Livingston DM, et al. Role of T-bet in commitment of TH1 cells before IL-12-dependent selection. Science. 2001;292(5523):1907-10.

23. Das J, Chen CH, Yang L, Cohn L, Ray P, Ray A. A critical role for NF-kappa B in GATA3 expression and $\mathrm{TH} 2$ differentiation in allergic airway inflammation. Nat Immunol. 2001;2(1):45-50.

24. Buck MD, O'Sullivan D, Pearce EL. T cell metabolism drives immunity. J Exp Med. 2015;212(9):1345-60.

25. Lu D, Sundstrom K, Sparen P, Fall K, Sjolander A, Dillner J, et al. Bereavement is associated with an increased risk of HPV infection and cervical cancer: an epidemiological study in Sweden. Cancer Res. 2016;76(3):643-51.

26. Kennedy B, Valdimarsdottir U, Sundstrom K, Sparen P, Lambe M, Fall K, et al. Loss of a parent and the risk of cancer in early life: a nationwide cohort study. Cancer Causes Control. 2014;25(4):499-506.

27. Antoni $\mathrm{MH}, \mathrm{Goodkin} \mathrm{K.} \mathrm{Host} \mathrm{moderator} \mathrm{variables} \mathrm{in} \mathrm{the} \mathrm{promotion} \mathrm{of}$ cervical neoplasia-I. Personality facets. J Psychosom Res. 1988;32(3):327-38.

28. Cesario SK, McFarlane J, Nava A, Gilroy H, Maddoux J. Linking cancer and intimate partner violence: the importance of screening women in the oncology setting. Clin J Oncol Nurs. 2014;18(1):65-73.

29. Coker AL, Bond S, Madeleine MM, Luchok K, Pirisi L. Psychosocial stress and cervical neoplasia risk. Psychosom Med. 2003;65(4):644-51.

30. Goodkin K, Antoni MH, Blaney PH. Stress and hopelessness in the promotion of cervical intraepithelial neoplasia to invasive squamous cell carcinoma of the cervix. J Psychosom Res. 1986;30(1):67-76.

31. Pereira DB, Antoni MH, Danielson A, Simon T, Efantis-Potter J, Carver CS, et al. Life stress and cervical squamous intraepithelial lesions in women with human papillomavirus and human immunodeficiency virus. Psychosom Med. 2003;65(3):427-34.

32. Tiersma ES, van der Lee ML, Garssen B, Peters AA, Visser AP, Fleuren GJ, et al. Psychosocial factors and the course of cervical intra-epithelial neoplasia: a prospective study. Gynecol Oncol. 2005;97(3):879-86.

33. Wilkerson JE, Bailey JM, Bieniasz ME, Murray SI, Ruffin MT. Psychosocial factors in risk of cervical intraepithelial lesions. J Women's Health (Larchmt). 2009:18(4):513-8.

34. Fang CY, Miller SM, Bovbjerg DH, Bergman C, Edelson MI, Rosenblum NG, et al. Perceived stress is associated with impaired T-cell response to HPV16 in women with cervical dysplasia. Ann Behav Med. 2008;35(1):87-96.

35. Richardson H, Kelsall G, Tellier P, Voyer H, Abrahamowicz M, Ferenczy A, et al. The natural history of type-specific human papillomavirus infections in female university students. Cancer Epidemiol Biomark Prevent. 2003;12(6):485-90.

36. Schulz P, Schlotz W, Becker P. Trierer Inventar zum chronischen stress (TICS). [Trier inventory for chronic stress (TICS)]. Göttingen: Hogrefe; 2004.

37. Asiaf A, Ahmad ST, Mohammad SO, Zargar MA. Review of the current knowledge on the epidemiology, pathogenesis, and prevention of human papillomavirus infection. Eur J Cancer Prevent. 2014;23(3):206-24.

38. Buysse DJ, Reynolds CF, Monk TH, Berman SR, Kupfer DJ. The Pittsburgh sleep quality index: a new instrument for psychiatric practice and research. Psychiatry Res. 1989;28(2):193-213.

39. Bassett SM, Lupis SB, Gianferante D, Rohleder N, Wolf JM. Sleep quality but not sleep quantity effects on cortisol responses to acute psychosocial stress. Stress. 2015;18(6):638-44.

40. Omisade A, Buxton OM, Rusak B. Impact of acute sleep restriction on cortisol and leptin levels in young women. Physiol Behav. 2010;99(5):651-6.

41. Pruessner JC, Kirschbaum C, Meinlschmid G, Hellhammer DH. Two formulas for computation of the area under the curve represent measures of total hormone concentration versus time-dependent change. Psychoneuroendocrinology. 2003;28(7):916-31. https://doi.org/10.1016/s0306-4530(02)00108-7.

42. Stalder T, Kirschbaum C, Kudielka BM, Adam EK, Pruessner JC, Wüst S, et al. Assessment of the cortisol awakening response: expert consensus guidelines. Psychoneuroendocrinology. 2016;63:414-32.
43. Hayes AF. Introduction to mediation, moderation, and conditional process analysis: a regression-based approach. New York: The Guilford Press; 2013.

44. Preacher KJ, Hayes AF. Asymptotic and resampling strategies for assessing and comparing indirect effects in multiple mediator models. Behav Res Methods. 2008;40(3):879-91.

45. Scott ME, Shvetsov YB, Thompson PJ, Hernandez BY, Zhu X, Wilkens LR, et al. Cervical cytokines and clearance of incident human papillomavirus infection: Hawaii HPV cohort study. Int J Cancer. 2013;133(5):1187-96.

46. Nickel C, Tanca S, Kolowos S, Pedrosa-Gil F, Bachler E, Loew TH, et al. Men with chronic occupational stress benefit from behavioural/psychoeducational group training: a randomized, prospective, controlled trial. Psychol Med. 2007;37(8):1141-9.

47. Waller J, McCaffery K, Kitchener H, Nazroo J, Wardle J. Women's experiences of repeated HPV testing in the context of cervical cancer screening: a qualitative study. Psycho-oncology. 2007;16(3):196-204.

48. Thangarajah F, Einzmann T, Bergauer F, Patzke J, Schmidt-Petruschkat S, Theune $M$, et al. Cervical screening program and the psychological impact of an abnormal pap smear: a self-assessment questionnaire study of 590 patients. Arch Gynecol Obstet. 2016;293(2):391-8.

\section{Publisher's Note}

Springer Nature remains neutral with regard to jurisdictional claims in published maps and institutional affiliations.
Ready to submit your research? Choose BMC and benefit from:

- fast, convenient online submission

- thorough peer review by experienced researchers in your field

- rapid publication on acceptance

- support for research data, including large and complex data types

- gold Open Access which fosters wider collaboration and increased citations

- maximum visibility for your research: over $100 \mathrm{M}$ website views per year

At BMC, research is always in progress.

Learn more biomedcentral.com/submissions 